\title{
Unusual Fatal Outcome Following Administration of a Combination of anti- PD1 and anti-CTLA4 in Metastatic Renal Cell Carcinoma: Liver Toxicity Case Report and a Literature Review
}

\author{
$\underline{\text { Nerina Denaro }}^{1}$, Ornella Garrone ${ }^{1}$, Marcella Occelli ${ }^{1}$, Elena Fea ${ }^{1}$, Cristina Granetto ${ }^{1}$, Marco Carlo Merlano ${ }^{2,3}$, Gianmauro Numico ${ }^{1}$ \\ ${ }^{1}$ Medical Oncology, St. Croce \& Carle University Teaching Hospital, Cuneo, Italy \\ ${ }^{2}$ Medical Oncology, IRCCS Candiolo, Turin, Italy \\ ${ }^{3}$ ARCO Foundation, Cuneo, Italy
}

Received: 13/05/2021

Accepted: 20/05/2021

Published: $17 / 06 / 2021$

\begin{abstract}
How to cite this article: Denaro N, Garrone O, Occelli M, Fea E, Granetto C, Merlano MC, Numico G. Unusual fatal outcome following administration of a combination of anti-PD1 and anti-CTLA4 in metastatic renal cell carcinoma: liver toxicity case report and a literature review. EJCRIM 2021;8: doi:10.12890/2021_002639.
\end{abstract}

Conflicts of Interests: ND, OG, MO, EF, CG and GN declare there are no conflicts of interest; MCM has worked as a consultant for MSD, Bristol-Myers Squibb and Merck.

Ethics' Approval: Ethics approval for use of the combination of anti-PD1 and anti-CTLA4 was obtained.

This article is licensed under a Commons Attribution Non-Commercial 4.0 License

\section{ABSTRACT}

Hepatic dysfunction, in the absence of liver metastases, occurs in 10-15\% of renal cell carcinoma (RCC) patients, while immune hepatitis due to anti-CTLA4 and anti-PD1 administration affects about 3-9\% and 0.7-1.8\% of treated patients, respectively. Liver toxicity following combination therapy (anti-CTLA4 and anti-PD1) is seen in $29 \%$ of patients overall and grade $3-4$ toxicity in $14 \%$ of patients.

Stauffer's syndrome is a rare para-neoplastic phenomenon associated with RCC and characterized by abnormal liver function tests, hepatosplenomegaly and histological changes consistent with non-specific hepatitis. We describe a case of RCC treated with anti-CTLA4 and antiPD1 therapy resulting in immediate liver toxicity and death after 2 months of progressive hepatic impairment. We hypothesize that high IL-6 levels due to Stauffer's syndrome might have contributed to immune-related hepatic failure.

\section{LEARNING POINTS}

- Consider Stauffer's syndrome in patients who develop liver toxicity unresponsive to immunotherapy.

- Evaluate IL-6 as high levels are seen in Stauffer's syndrome patients undergoing immunotherapy.

- Consider taking a liver biopsy to assess the severity of liver injury.

\section{KEYWORDS}

Hepatitis, hepatic failure, immunotherapy, renal carcinoma

\section{CASE DESCRIPTION}

In June 2019, a 56-year-old woman without a relevant medical history underwent right renal tumourectomy. Histology findings demonstrated clear cell renal cell carcinoma (RCC), sarcomatoid type, grade 4 (Fuhrman), free margins, pT1b Nx M0. On 26 July after a multidisciplinary team discussion (MTD), follow-up with chest and abdominal CT scans within 1 month was scheduled.

In August 2019, the patient was admitted to the emergency department for neurological symptoms. A brain CT scan showed a left frontal lesion. She was admitted to the neurology department and started steroid treatment. A whole-body CT scan confirmed progressive disease with mediastinal node involvement and two enlarged abdominal nodes (one perirenal and one close to the right iliac vessels). 
Steroid therapy during hospitalization resulted in improvement.

In August 2019, the MTD advised radical single brain metastasis excision followed by systemic therapy. The patient underwent brain surgery. Histology showed epithelial neoplasia with clear cell aspects and large necrotic areas, immuno-morphologically consistent with secondary RCC.

In October 2019, the patient started combination immunotherapy with ipilimumab and nivolumab. After 1 week, liver toxicity was reported (grade 2 on aspartate aminotransferase (AST) and alanine transaminase (ALT), and grade 3 on total bilirubin (TB) and direct bilirubin (DB)). The patient then underwent a liver ultrasound (Fig. 1) which was negative for metastasis. She was started on oral prednisone $25 \mathrm{mg}(1$ capsule) three times a day.
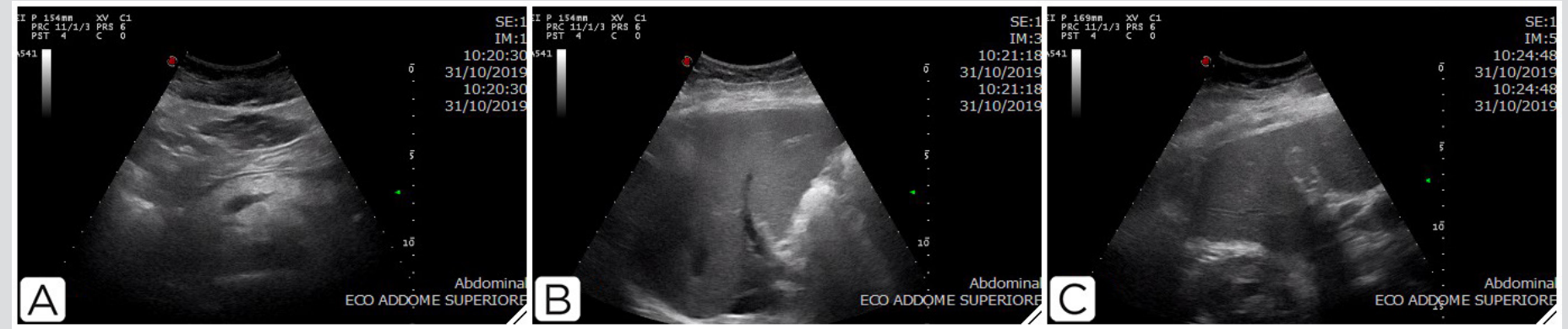

Figure 1. Ultrasound scan on 31 October 2019

On 4 November, due to progressive liver impairment, the patient started methylprednisolone at $2 \mathrm{mg} / \mathrm{kg}$. Alternative causes for the liver impairment were investigated and excluded. Tests for hepatitis A, B and C infection, cytomegalovirus and immune hepatitis were performed and resulted negative.

Budd-Chiari syndrome was excluded because neither imaging or coagulation status were suggestive for the disease.

Blood analysis demonstrated worsening liver toxicity. We considered the liver failure was due to immune-related toxicity and increased the steroid dose. The patient was admitted to the oncology ward to start intravenous high-dose steroids according to international guideline ${ }^{[1-3]}$. The patient's medication included $2 \mathrm{mg} / \mathrm{kg}$ methylprednisolone (daily dose $80 \mathrm{mg} \times 2$ ), euthyrox $125 \mu \mathrm{mg}$, levetiracetam $500 \mathrm{mg} \times 2$, furosemide $20 \mathrm{mg} \times 2$, omeprazole $20 \mathrm{mg} \times 2$ in the evening, delorazepam $8 \mathrm{mg}$ in the evening, and cetirizine $10 \mathrm{mg}(1$ capsule $) \times 2$. The blood results during treatment are given in Table 1.

In November 2019, the patient underwent an abdominal CT scan (Fig. 2A) which showed a slightly enlarged liver without focal lesions. Liver enzymes continued to increase without impairment in coagulation function. The patient maintained a fair general condition until the beginning of December 2019.
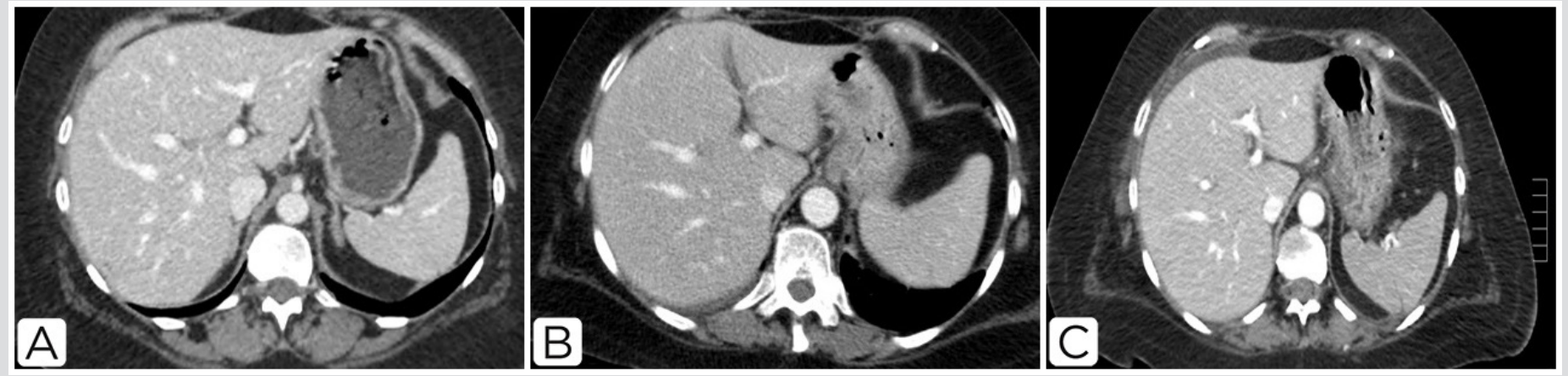

Figure 2. CT scans on 11 November 2019 (A), 17 December 2019 (B) and 23 December 2019 (C)

At the end of November, the patient started mycophenolate $100 \mathrm{mg}$ twice daily and underwent liver ultrasound plus-guided biopsy (Fig. 3). Liver enzyme trends are shown in Fig. 4. The histology report noted hepatic tissue with cytoplasmic and intracanalicular cholestasis, sinusoid ectasia and histiocytosis, tissue macrophage or dendritic cell aggregation, and microfoci of liver necrosis in single or a few cells. 
There was no evidence of endotheliosis.

On 11 December, the patient started the third-line immunosuppressive therapy with tacrolimus 2 mg daily. On 17 December, she underwent an abdominal CT scan (Fig. 2B) which showed progressive disease but no liver involvement. The patient's condition deteriorated and she died on 27 December. Her relatives did not consent to an autopsy.
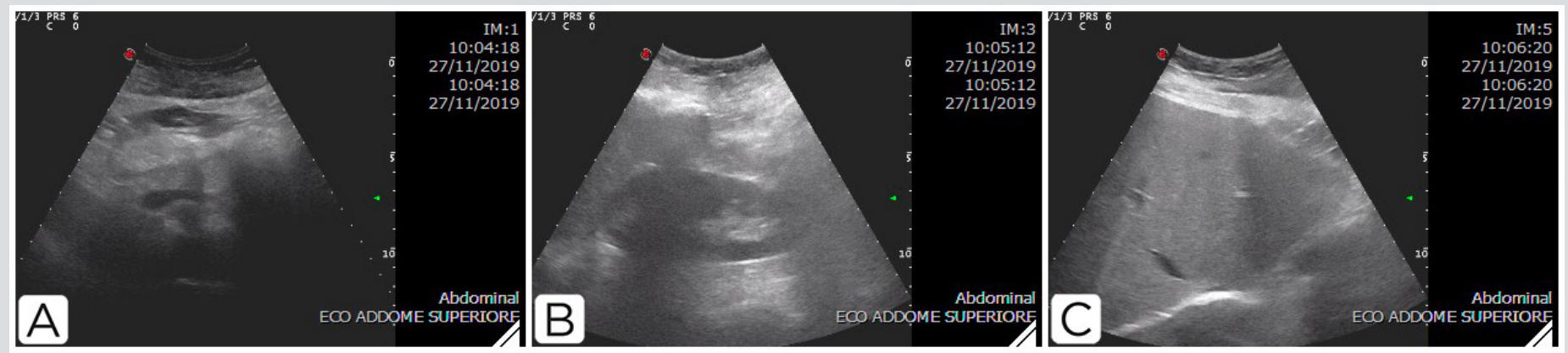

Figure 3. Ultrasound scan on 27 November 2019

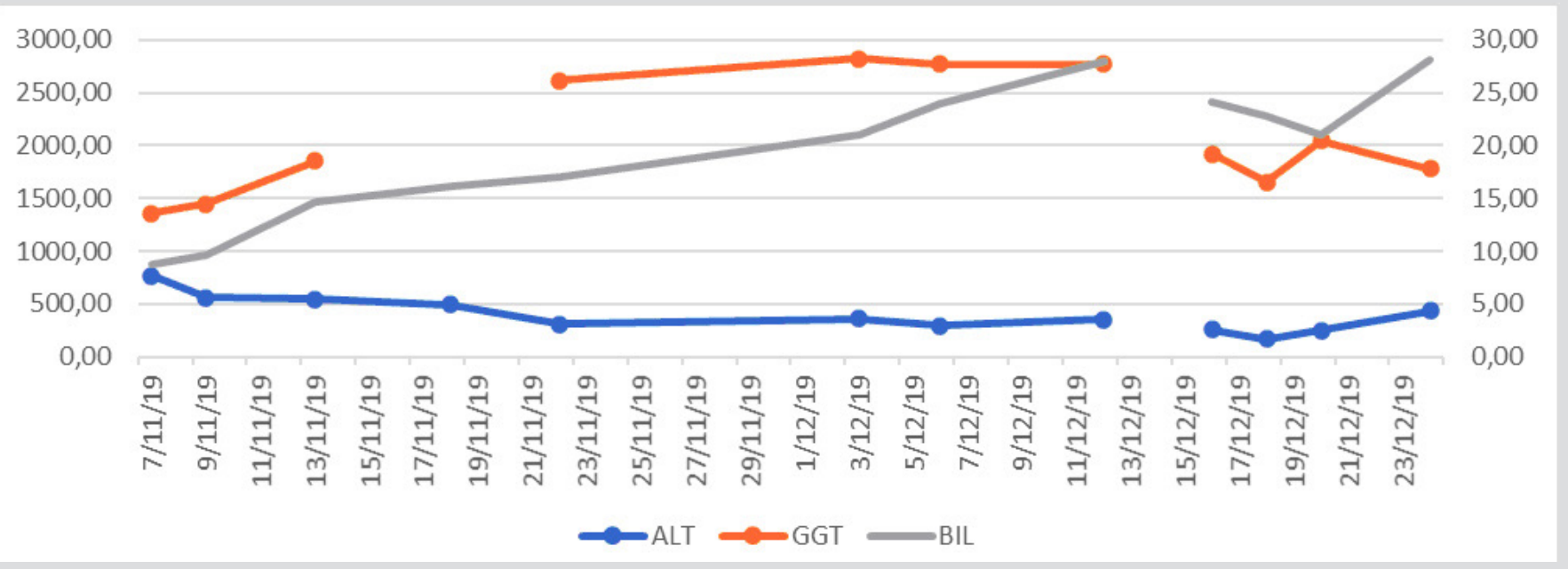

Figure 4. Liver enzyme trends.

ALT, alanine transaminase (U/l); BIL, bilirubin (mg/dl); GGT, gamma-glutamyl transpeptidase (U/I)

\section{DISCUSSION}

Immunotherapy is an important treatment for cancer. However, its use is associated with a spectrum of adverse effects (immune-related adverse events, irAEs) related to the drugs' mechanisms of action. The adverse effects most commonly affect the skin, gastrointestinal tract, lungs and endocrine glands. Hepatic irAEs are uncommon and little is known about their pathogenesis and management as they are quite rare, at least with monotherapy. Recently, a pooled analysis in melanoma patients showed that immune hepatitis due to anti-CTLA4 and anti-PD1 treatment affects about 3-9\% and $0.7-1.8 \%$ of patients, respectively, and causes grade $3-4$ hepatic toxicity in up to $14 \%$ of patients ${ }^{[1]}$. Currently, guidelines recommend high-dose steroids (from 1 to $2 \mathrm{mg} / \mathrm{kg} /$ day) for grade 3-4 or persistent grade 2 hepatitis (more than 1-2 weeks). If there is no response to corticosteroids within 2-3 days, mycophenolate should be considered ${ }^{[2-4]}$.

Zarrabi et al. reported a case of RCC in which hyper-bilirubinaemia was unrelated to irAEs but correlated with Stauffer's syndrome and benefited from renal surgery ${ }^{[5]}$. Stauffer's syndrome is characterized by cholestatic liver dysfunction in the absence of underlying hepatobiliary disease (elevated TB, DB and alkaline phosphatase). It is estimated that hepatic dysfunction, in the absence of liver metastases, occurs in 10-15\% of RCC cases, but it is difficult to estimate the incidence of Stauffer's syndrome as the literature is mainly confined to case reports ${ }^{[6]}$. Stauffer's syndrome is associated with an unfavourable prognosis ${ }^{[7]}$. In 2018, having considered knowledge accumulated since 1961 when the syndrome was first described, Fontes-Sousa et al. proposed the use of a broader designation, that is 'paraneoplastic intrahepatic cholestasis', and new diagnostic criteria, which are given in Table $2^{[6]}$. 


\begin{tabular}{|l|l|l|l|l|l|l|l|l|l|l|l|}
\hline & PLT (//l) & $\begin{array}{l}\text { WBC } \\
\left(\times 10^{3} / \text { Ill) }\right.\end{array}$ & HB (g/dl) & $\begin{array}{l}\text { UREA } \\
\text { (mg/dl) }\end{array}$ & $\begin{array}{l}\text { CREA } \\
\text { (mg/dl) }\end{array}$ & INR & AST (U/I) & ALT (U/I) & GGT (U/I) & BIL (mg/dl) & ALP (U/I) \\
\hline $16 / 06 / 2019$ & 248 & 6.2 & 9 & & & & & & & & \\
\hline $05 / 11 / 2019$ & 183 & 15.6 & 11.1 & & & 1.02 & & & & & \\
\hline $07 / 11 / 2019$ & & & & & & & 1142 & 765 & 1359 & 8.8 & \\
\hline $09 / 11 / 2019$ & & & & & & & 1069 & 558 & 1448 & 9.7 & \\
\hline $13 / 11 / 2019$ & & & & & & & 1220 & 541 & 1852 & 14.6 & \\
\hline $18 / 11 / 2019$ & 195 & 3.19 & 9.1 & 18 & 0.52 & 1.05 & 1150 & 491 & & 16.1 & \\
\hline $22 / 11 / 2019$ & 185 & 9.87 & 9.0 & 17 & 0.43 & 1.13 & 992 & 311 & 2620 & 17.1 & 450 \\
\hline $03 / 12 / 2019$ & 147 & 5.87 & 8.8 & & & 1.03 & 847 & 356 & 2827 & 21 & \\
\hline $06 / 12 / 2019$ & & & & & & 1.08 & 775 & 296 & 2773 & 24 & \\
\hline $12 / 12 / 2019$ & 94 & 4.22 & 8.1 & & 0.35 & & 751 & 352 & 2774 & 28 & \\
\hline $14 / 12 / 2019$ & 93 & 5.16 & 8.3 & & & 1.16 & & & & & \\
\hline $16 / 12 / 2019$ & 167 & 6.75 & 8.5 & & 0.61 & 1.10 & 613 & 254 & 1924 & 24.1 & 725 \\
\hline $18 / 12 / 2019$ & 146 & 5.9 & 9.3 & 31 & 0.4 & 1.05 & 439 & 167 & 1658 & 22.8 & 718 \\
\hline $20 / 12 / 2019$ & 92 & 8.3 & 7.6 & & & 1.05 & 451 & 247 & 2048 & 21 & \\
\hline $24 / 12 / 2019$ & 97 & 7.09 & 8.9 & & & 1 & 606 & 432 & 1778 & 28.1 & \\
\hline $25 / 12 / 2019$ & 68 & 5.75 & 7.9 & 49 & 0.6 & & & & & & \\
\hline $26 / 12 / 2019$ & 52 & 4.91 & 10.3 & & & & & & & & \\
\hline
\end{tabular}

Table 1. Blood test results Normal ranges: alanine transaminase (ALT) to 49 U/l; alkaline phosphatase (ALP) 38-126 U/l; aspartate aminotransferase (AST) to 34 U/l; bilirubin, total (BIL) 0.3-1.2 mg/dl; creatinine (CREA) 0.4-1.10 mg/dl; gamma-glutamyl transpeptidase (GGT) to 49 U/l; haemoglobin (HB) 12-16 g/dl; platelets (PLT) 150-400,000/ul; urea (UREA) 9.0-23.0

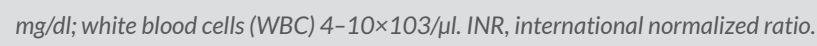

\begin{tabular}{|c|c|}
\hline Criterion & Features \\
\hline 1 & Histologically proven diagnosis of malignancy, most notably renal cell carcinoma \\
\hline 2 & $\begin{array}{l}\text { Mandatory liver dysfunction or cholestasis, including prolonged thrombin time; jaundice, pruritus or } \\
\text { urinary hyperpigmentation may be present }\end{array}$ \\
\hline 3 & $\begin{array}{l}\text { No evidence or suspicion of liver metastasis or mass effect on liver or biliary ducts, such as direct tumour } \\
\text { or nodal disease with hepatic invasion or compression }\end{array}$ \\
\hline 4 & Hepatosplenomegaly may be present \\
\hline 5 & $\begin{array}{l}\text { The alterations are reversible in nature, and expected to normalize in the weeks to months after treatment } \\
\text { of the primary tumour }\end{array}$ \\
\hline 6 & In the metastasized setting (excluding liver), the alterations may be reversible with systemic therapy \\
\hline 7 & $\begin{array}{l}\text { Other causes of intrahepatic cholestasis should be excluded. Liver biopsy may be necessary for accurate } \\
\text { differential diagnosis }\end{array}$ \\
\hline
\end{tabular}

Table 2. Stauffer's syndrome and intrahepatic cholestasis paraneoplastic criteria 
To our knowledge, this is the first report of grade 5 hepatic toxicity following administration of a combination of anti-PD1 and anti-CTLA4 in a patient with possible latent Stauffer's syndrome. In our patient, Stauffer's syndrome was not described at the onset of RCC; however, after the first dose of combination immunotherapy, she developed hepatic toxicity refractory to immunosuppressive therapy. We hypothesize that a variant of Stauffer's syndrome may have caused the clinical features and that immunotherapy worsened the hepatic failure. In our patient both endotheliosis and histiocytosis was described on liver histology without liver metastasis or hepatitis. Current available guidelines for irAEs were followed. Of note, Fontes-Sousa et al. in their review did not find that immunotherapy affected hepatic symptoms in Stauffer's syndrome ${ }^{[6]}$.

In retrospect, we should have measured IL-6 levels in our patient, because we later hypothesized that IL-6 might have had a role in the pathophysiology of checkpoint inhibitor-associated hepatotoxicity and Stauffer's syndrome. The central role of IL-6 in Stauffer's syndrome pathogenesis and in immunotherapy responses supports this conclusion.

The case report confirms the role of liver biopsy in reaching the diagnosis and evaluating the severity of liver injury as well as establishing the absence of metastases or underlying liver disease, and demonstrates the need for patient-oriented management, which could eventually avoid unnecessary systemic corticosteroid treatment. Our patient received higher-dose but ultimately useless steroid treatment and was refractory to all hepatitis treatment administered. Also, our patient did not have predictors of immune events, according to the Kartolo et al. classification, except for use of anti-CTLA4 inhibitors ${ }^{[7]}$. We should have considered earlier the possibility of a cause other than immunotoxicity and we recommend a liver biopsy should be taken earlier (within 2 weeks). A flow chart for the management of hepatic failure after the administration of combination therapy is provided in Table 3.

\begin{tabular}{|c|l|}
\hline Criterion & Features \\
\hline 1 & Screening for viral, bacterial and drug-related causes of hepatitis \\
\hline 2 & Steroid administration according current immune-related adverse event (irAE) guidelines \\
\hline 3 & Liver biopsy \\
\hline 4 & IL-6 test (differential diagnosis of Stauffer's syndrome and prognosticator) \\
\hline 5 & $\begin{array}{c}\text { Consider surgery or other targeted therapy if immunosuppressive therapy fails (including mycophenolate } \\
\text { and plasma exchange) }\end{array}$ \\
\hline
\end{tabular}

Table 3. Proposed liver evaluation in renal cell carcinoma treated with combination immunotherapy

\section{REFERENCES}

1. Sznol M, Ferrucci PF, Hogg D, Atkins MB, Wolter P, Guidoboni M, et al. Pooled analysis safety profile of nivolumab and ipilimumab combination therapy in patients with advanced melanoma. J Clin Oncol 2017;35(34):3815-3822

2. Trinh S, Le A, Gowani S, La-Beck N. Management of immune-related adverse events associated with immune checkpoint inhibitor therapy: a minireview of current clinical guidelines. Asia Pac J Oncol Nurs 2019;6(2):154-160.

3. Brahmer JR, Lacchetti C, Thompson JA. Management of immune-related adverse events in patients treated with immune checkpoint inhibitor therapy: American Society of Clinical Oncology clinical practice guideline summary. J Oncol Pract 2018;14(4):247-249.

4. De Martin E, Michot JM, Papouin B, Champiat S, Mateus C, Lambotte O, et al. Characterization of liver injury induced by cancer immunotherapy using immune checkpoint inhibitors. J Hepatol 2018;68(6):1181-1190. doi: 10.1016/j.jhep.2018.01.033

5. Zarrabi K, Masic S, Schaefer C, Bartel MJ, Kutikov A, Zibelman M, et al. Neoadjuvant checkpoint inhibition in renal cell carcinoma associated Stauffer's syndrome. Urol Case Rep 2020;29:101077.

6. Fontes-Sousa M, Magalhães H, da Silva FC, Maurício MJ. Stauffer's syndrome: a comprehensive review and proposed updated diagnostic criteria. Urol Oncol 2018;36(7):321326. doi: 10.1016/j.urolonc.2018.01.019

7. Kartolo A, Sattar J, Sahai V, Baetz T, Lakoff JM. Predictors of immunotherapy-induced immune-related adverse events. Curr Oncol 2018;25(5):e403-e410. doi: 10.3747/ co.25.4047 